\title{
Angiographic and Clinical Outcomes of a Pharmacokinetic Study of Sirolimus-Eluting Stents Lesson From Restenosis Cases
}

\author{
Masato Nakamura, MD; Masamichi Wada, MD; Hisao Hara, MD; Ken Kozuma, MD*; \\ Yoritaka Otsuka, MD**; Shunichi Miyazaki, MD**
}

\begin{abstract}
Background A pharmacokinetic (PK) study was conducted to evaluate sirolimus-eluting stents (SES) in Japanese people, representing the first clinical trial of the use of drug-eluting stents in Japan.

Methods and Results The PK study was conducted in 20 patients with 30 lesions treated with sirolimuscoated BX Velocity stents. All lesions were treated with a single SES $(3 \times 18 \mathrm{~mm})$. Angiographic follow-up was performed at 8 months after SES implantation, and the clinical outcomes were evaluated at 1 year in all cases. All procedures were successful, and all patients were discharged without any adverse cardiac events. The total restenosis rate was 10\% (3 lesions) and target vessel revascularization was performed in those 3 cases (15\%). Restenoses occurred at the proximal and distal stent margins. Intravascular ultrasound examination of restenosis cases revealed abundant plaque burden at the stent edges even though the luminal area was preserved.

Conclusions The sirolimus-eluting BX Velocity stent is safe and useful for Japanese patients with coronary artery disease. However, restenosis at proximal stent edge seems to be a problem. (Circ J 2005; 69: 1196-1201)
\end{abstract}

Key Words: Drug eluting stent; Intravascular ultrasound; Restenosis

$\mathbf{R}$ estenosis is the main limitation of percutaneous coronary intervention (PCI), but the introduction of drug-eluting stents (DES) is a breakthrough development. Clinical trials of DES have reported a markedly decreased rates of restenosis and revascularization compared with bare metal stents (BMS), ${ }^{1-6}$ but the efficacy of DES in Japanese people is still unclear.

Prior to getting approval for the sirolimus-eluting stent (SES: Cypher stent, Cordis Corp, a Johnson\&Johnson Company) in Japan, a pharmacokinetic (PK) study evaluating the PKs of sirolimus in Japanese patients and the 1-year clinical outcomes was conducted?

\section{Methods}

\section{Study Subjects}

The PK study was the first clinical trial of the SES in Japan, and its aim was to evaluate the PKs of sirolimus in Japanese patients and to evaluate early and late clinical outcomes. It was a nonrandomized open trial, comprising 20 patients with 30 lesions who underwent implantation of SES. The patients were all symptomatic and/or had functionally significant de novo native coronary artery disease. Patients were excluded from this study if they had (1) acute myocardial infarction, (2) unstable angina (Braunwald III

(Received June 2, 2005; revised manuscript received July 8, 2005; accepted July 21, 2005)

Division of Cardiovascular Medicine, Toho University Ohashi Medical Center, *Department of Cardiology, Teikyo University School of Medicine, Tokyo and **Department of Cardiology, National Cardiovascular Center, Suita, Japan

Mailing address: Masato Nakamura, MD, Division of Cardiovascular Medicine, Toho University Ohashi Medical Center, 2-17-6 Ohashi, Meguro-ku, Tokyo 153-8515, Japan. E-mail: masato@oha.toho-u.ac. jp
B, III C), (3) bifurcation lesions, (4) ostial lesions, (5) left main trunk disease, (6) severe calcification, (7) chronic total occlusion, (8) renal dysfunction (serum creatinine $>3.0 \mathrm{mg} / \mathrm{dl}$ ) or liver dysfunction, (9) proximal reference diameter $<2.5 \mathrm{~mm}$, (10) allergy to antiplatelet drugs, heparin and sirolimus, (11) extreme bending lesion, (12) left ventricular ejection fraction $<25 \%$, (13) cardiac transplantation or (14) another stent within $5 \mathrm{~mm}$ of target lesion. The ethical committee at each participating institute approved the protocols, and all patients gave written informed consent.

\section{Procedure}

Before angioplasty, heparin was given intravenously and titrated to maintain an active clotting time more than $250 \mathrm{~ms}$. All patients received a BX velocity stent $(3 \times$ $18 \mathrm{~mm}$ ) loaded with a total sirolimus doses of $150 \mathrm{\mu} \mathrm{g}$. All lesions were pre-dilated before stent implantation and adequate post dilatation was performed. All patients received aspirin $(200 \mathrm{mg} /$ day indefinitely) and ticlopidine $(200 \mathrm{mg} /$ day) from 3 days before the procedure and continued daily for 3 months after SES implantation.

\section{Quantitative Coronary Angiography (QCA)}

Detailed QCA parameters of the target lesion were evaluated before and after the procedure and at the time of angiographic follow-up using a computer-based system (QCA-CMS 5.1, MEDIS, Leiden, the Netherlands) and an independent observer at Toho University Ohashi Medical Center. This QCA software allows us to also measure automatically all parameters for $5 \mathrm{~mm}$ proximal and distal to the stented segment. At the follow-up analysis, in-stent restenosis (ISR) was defined as a diameter stenosis $>50 \%$ within the stent segment. In-segment restenosis was defined as $>50 \%$ either within the stented segment or within 
Table 1 Patients Characteristics

\begin{tabular}{lc}
\hline \hline$n$ & 20 \\
Age (years) & $64.2 \pm 9.9$ \\
Height $(\mathrm{cm})$ & $162.1 \pm 8.3$ \\
Weight $(\mathrm{kg})$ & $65.3 \pm 12.6$ \\
Male, $n(\%)$ & $16(80)$ \\
Risk factors & \\
Hypertension, $n(\%)$ & $16(60)$ \\
Hyperlipidemia, $n(\%)$ & $11(55)$ \\
Diabetes mellitus, $n(\%)$ & $12(60)$ \\
Smoking, $n(\%)$ & $13(65)$ \\
Obesity, $n(\%)$ & $7(35)$ \\
Target vessel, $n(\%)$ & \\
LAD & $12(40)$ \\
LCX & $6(20)$ \\
RCA & $12(40)$ \\
Ejection fraction $(\%)$ & $61.0 \pm 11.2$ \\
Previous MI, $n(\%)$ & $3(15)$ \\
Previous PCI, $n(\%)$ & $7(35)$ \\
Previous CABG, $n(\%)$ & 0 \\
Lesion type $($ ACC/AHA), $n(\%)$ & 30 \\
A/B1 & $24(80)$ \\
B2/C & $6(20)$ \\
\hline
\end{tabular}

LAD, left anterior descending coronary artery; $L C X$, left circumflex coronary artery; RCA, right coronary artery; $M I$, myocardial infarction; $P C I$, percutaneous coronary intervention; $C A B G$, coronary artery bypass grafting; ACC, American College of Cardiology; AHA, American Heart Association.

$5 \mathrm{~mm}$ proximal or distal to the stent segment. In-stent late lumen loss was defined as the difference between the minimal luminal diameter (MLD) immediately after the procedure and the MLD at follow-up.

\section{Intravascular Ultrasound (IVUS) Studies}

IVUS studies conducted at the end of the procedure and at the time of follow-up used a 30- or 40-MHz transducertipped catheter (Ultra-cross 3.2Fr, Atlantis SR3.0Fr and Atlantis SR Plus 2.5Fr, Boston Scientific, Sunnyvale, CA, USA). IVUS images were acquired during motorized pullback at a constant speed of $0.5 \mathrm{~mm} / \mathrm{s}$ and were recorded on VHS video tape. Acquisition of IVUS images was started at least $1 \mathrm{~cm}$ distal to the stent and ended at least $1 \mathrm{~cm}$ proximal to stent.

\section{Results}

\section{Baseline Characteristics}

The patient and lesion profiles are summarized in Table 1. The mean age was $64.2 \pm 9.9$ years, and a high prevalence of patients with diabetes mellitus.

\section{Procedural Data}

The mean lesion length and reference diameter were $12.1 \pm 3.8 \mathrm{~mm}$ and $2.67 \pm 0.58 \mathrm{~mm}$, respectively. Stents were deployed with a mean pressure of $14.7 \pm 3.7 \mathrm{mmHg}$, and dilated by post dilatation balloon with a pressure of $15.4 \pm$ $3.3 \mathrm{mmHg}$. Procedural success was achieved in all patients, and all cases were discharged without any adverse cardiac events.

\section{Angiographic Follow-up Data}

Angiographically analyzed data are shown in Table 2. In-segment and ISR per lesion occurred in $3(10 \%)$ and 2 lesions $(6.6 \%)$, respectively. Focal-type restenosis occurred at the proximal edge of the stent (Fig 1) in 1 case, and totalocclusion-type ISR occurred in 1 case that involved 2 SES
Table 2 Angiographic Data

\begin{tabular}{lc}
\hline \hline In-stent analysis & \\
Reference vessel (mm) & $2.67 \pm 0.58$ \\
MLD (mm) & $0.99 \pm 0.48$ \\
$\quad$ Pre & $2.73 \pm 0.47$ \\
Post & $2.46 \pm 0.66$ \\
Follow-up & \\
\%DS & $62.8 \pm 16.4$ \\
Pre & $12.1 \pm 5.71$ \\
Post & $18.9 \pm 17.6$ \\
Follow-up & $0.27 \pm 0.69$ \\
Late loss & \\
In-segment analysis & $2.67 \pm 0.58$ \\
Reference vessel (mm) & \\
MLD (mm) & $0.99 \pm 0.48$ \\
Pre & $2.59 \pm 1.64$ \\
Post & $2.10 \pm 0.70$ \\
Follow-up & \\
\% DS & $62.8 \pm 16.4$ \\
Pre & $25.2 \pm 12.1$ \\
Post & $31.5 \pm 21.5$ \\
Follow-up & $0.50 \pm 1.52$ \\
Late loss & $0.17 \pm 0.75$ \\
Late loss at proximal margin & $0.21 \pm 0.63$ \\
Late loss at distal margin & \\
Angiographic restenosis & $2(6.6 \%)$ \\
In-stent & $3(10 \%)$ \\
In-segment & \\
\hline
\end{tabular}

Continuous variables are presented as mean (SD).

Late loss, difference between MLD at 8months and MLD after procedure. $M L D$, minimum lumen diameter; $D S$, diameter stenosis.

implantations (Fig 2). Late luminal loss was $0.27 \pm 0.69 \mathrm{~mm}$ in-stent, and $0.50 \pm 1.52 \mathrm{~mm}$ in-segment.

\section{One-Year Outcome}

Ticlopidine was discontinued in 2 cases because of rash and abnormal laboratory test values, but with no adverse events. At 1-year follow-up, none of the cases was complicated by stent thrombosis. There were 3 major adverse cardiac events $(15 \%)$ during the follow-up period, but no deaths or myocardial infarctions, and only 1 target lesion revascularization (TLR) or target vessel revascularization. Two cases complicated by angiographic restenoses were successfully treated by additional stenting at 10 months, and another case had target vessel revascularization of the proximal stent segment at 1 year. These cases were asymptomatic, and revascularization was indicated by a positive stress test.

\section{Insights of the TLR Cases From IVUS and Angiographic Examinations}

Stent Edge Restenosis Case IVUS assessment after DES deployment revealed that the percent plaque area (\%PA) at the proximal stent edge was $64.8 \%$, and plaque area had increased from $10.5 \mathrm{~mm}^{2}$ to $13.6 \mathrm{~mm}^{2}$ (Figs 4,5).

Total-Occlusion-Type ISR Case SES implantation was performed for a $99 \%$ stenosed proximal right coronary artery (RCA) lesion and a 75\% mid RCA lesion (Fig 3). Follow-up angiography revealed that the RCA was totally occluded in the segment proximal to the SES. Angiography after recanalization of the occluded proximal RCA with a small balloon $(1.5 \mathrm{~mm}$ in diameter) revealed that the instent segment was fully patent. A side branch within the DES was open even though RCA was totally occluded and not being filled by collateral flow from the left coronary artery (Fig 3). IVUS examination revealed that the plaque 


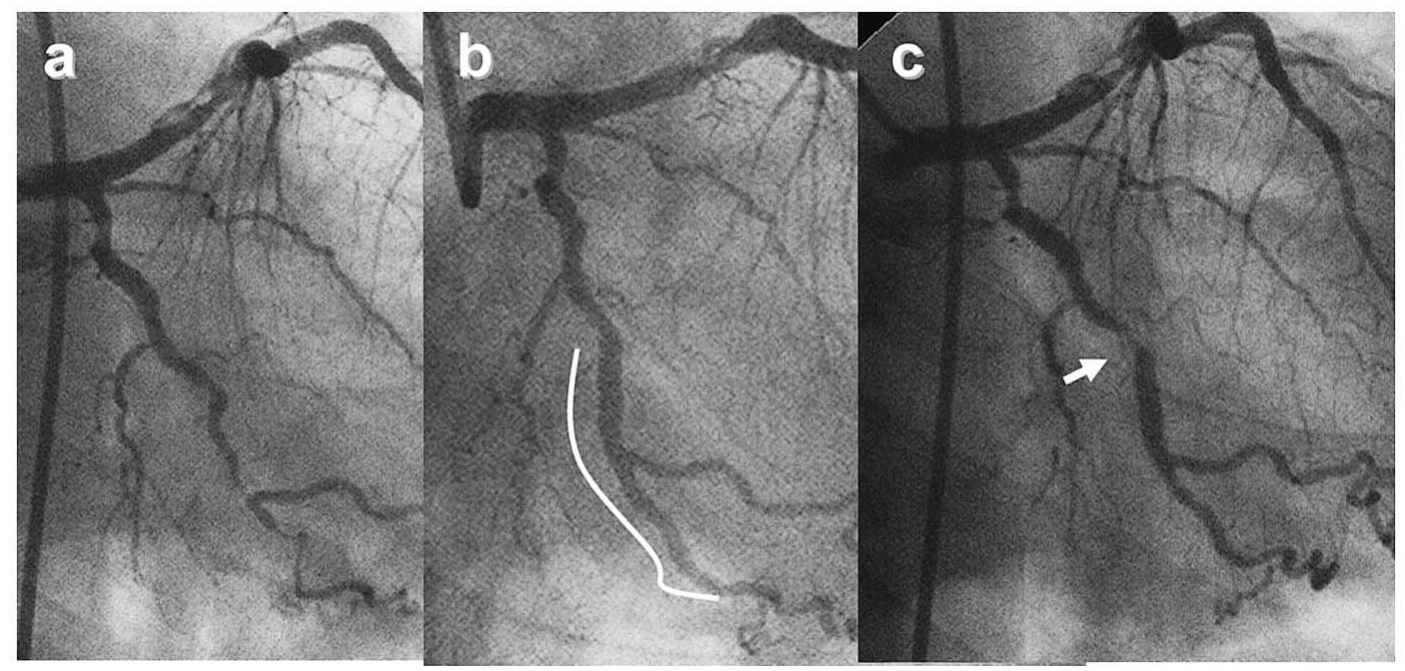

Fig 1. Angiograms of proximal stent edge restenosis after the procedure (Right) and at follow-up (Left). There is focal restenosis (arrow) at the proximal stent edge. White line represents sirolimus-eluting stent implanted in the left circumflex artery.

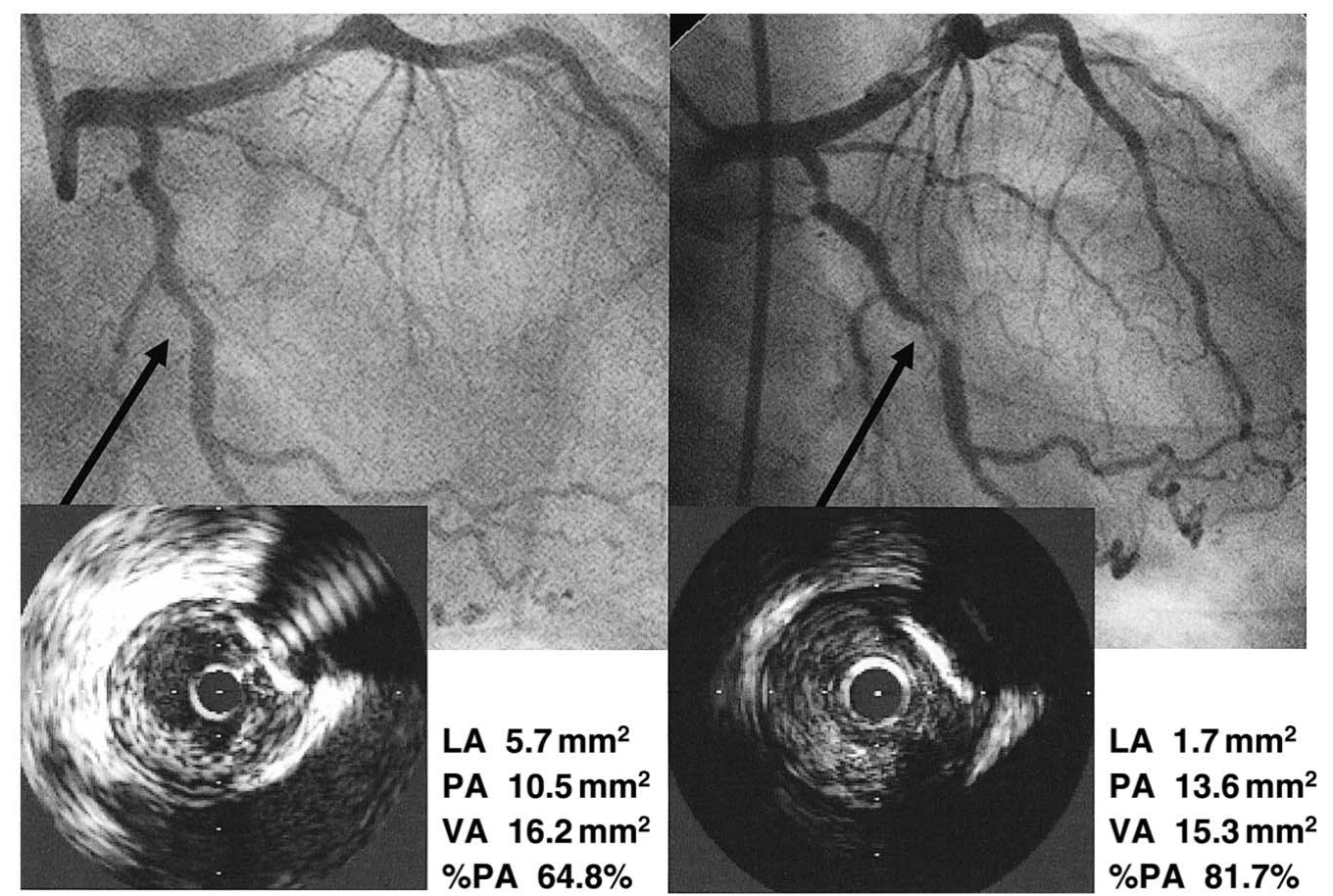

Fig 2. Intravascular ultrasound images of proximal stent edge restenosis after the procedure and at follow-up. After stent deployment, the plaque area (PA) at the stent edge is $10.5 \mathrm{~mm}^{2}$ and it increased to $13.6 \mathrm{~mm}^{2}$ at the end of the follow-up period. LA, lumen area; VA, vessel area.

burden in the proximal stent segment was more than $50 \%$ (Fig6). The serial IVUS examination also demonstrated that luminal narrowing at the stent edge, not intimal hyperplasia, was contributing to the narrowing of the vessel area (Fig 7).

\section{Discussion}

This study is the first Japanese experience with implantation of the sirolimus-coated BX Velocity stent. We report (1) procedural success in all cases without any adverse event during hospitalization; (2) restenosis at the proximal and distal margins of the SES; (3) the presence of a large plaque burden adjacent to the stent on IVUS assessment might be a predictor of stent edge restenosis.

\section{Edge Restenosis}

Restenosis occurred not within the stent but at its margins, which concurs with the findings of the Sirius trial, which reported that in-segment restenosis occurred at a higher frequency at the proximal margin of the stent than at the distal margin or in the body of the stent? It has been reported that $87 \%$ of the restenoses were focal type, and that total occlusion was extremely rare; ${ }^{2}$ However, in the 


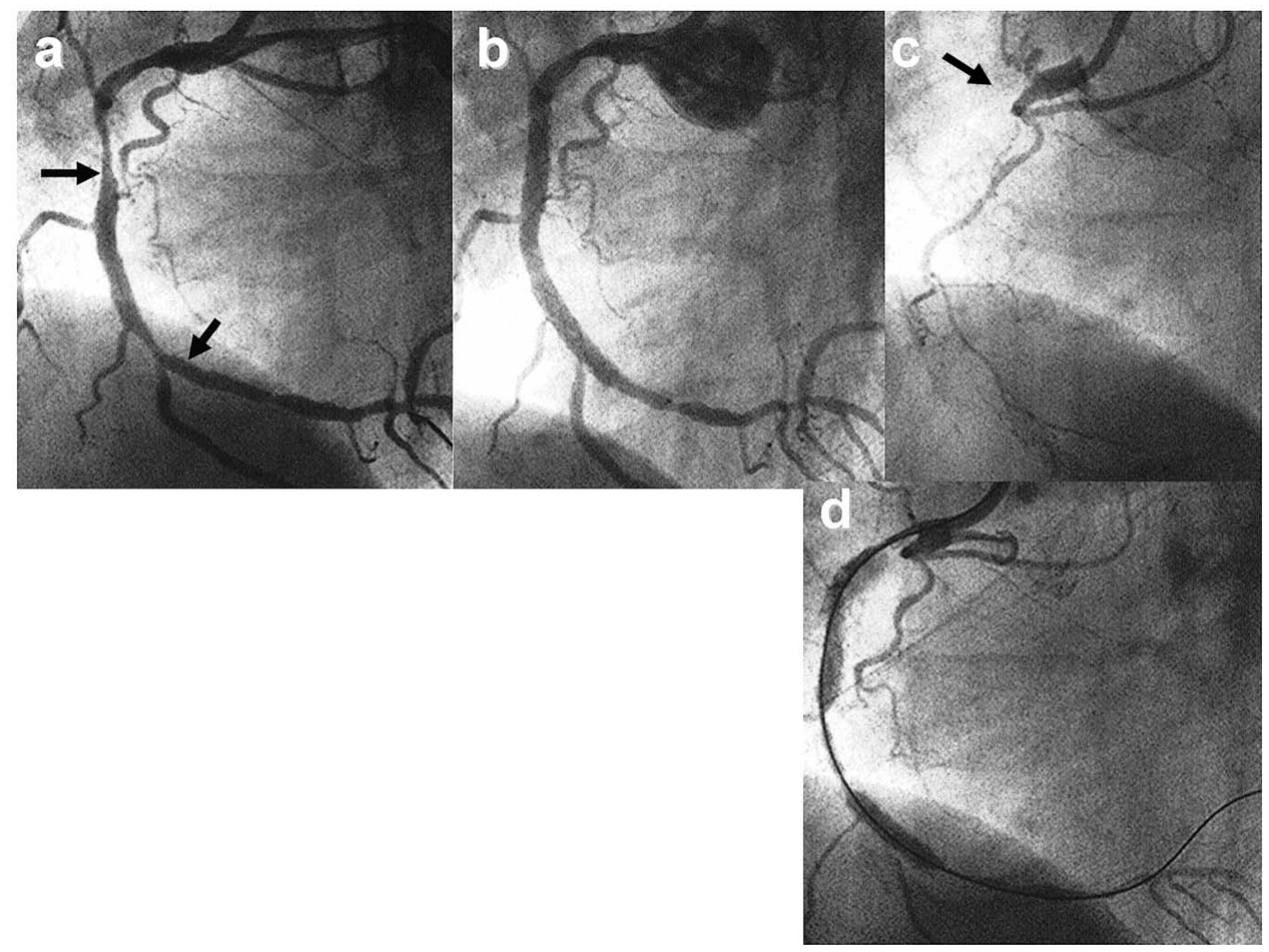

Fig 3. Angiograms of the case of total-occlusion-type in-stent restenosis before (a) and after the procedure (b), at follow-up (c), and after recanalization (d). Follow-up angiography revealed occlusion of the right coronary artery proximal to the stented segment. The angiogram after recanalization (d) shows that the lumen and side branch of the sirolimuseluting stent are open. There is a "candy-wrapper appearance" to this angiogram.

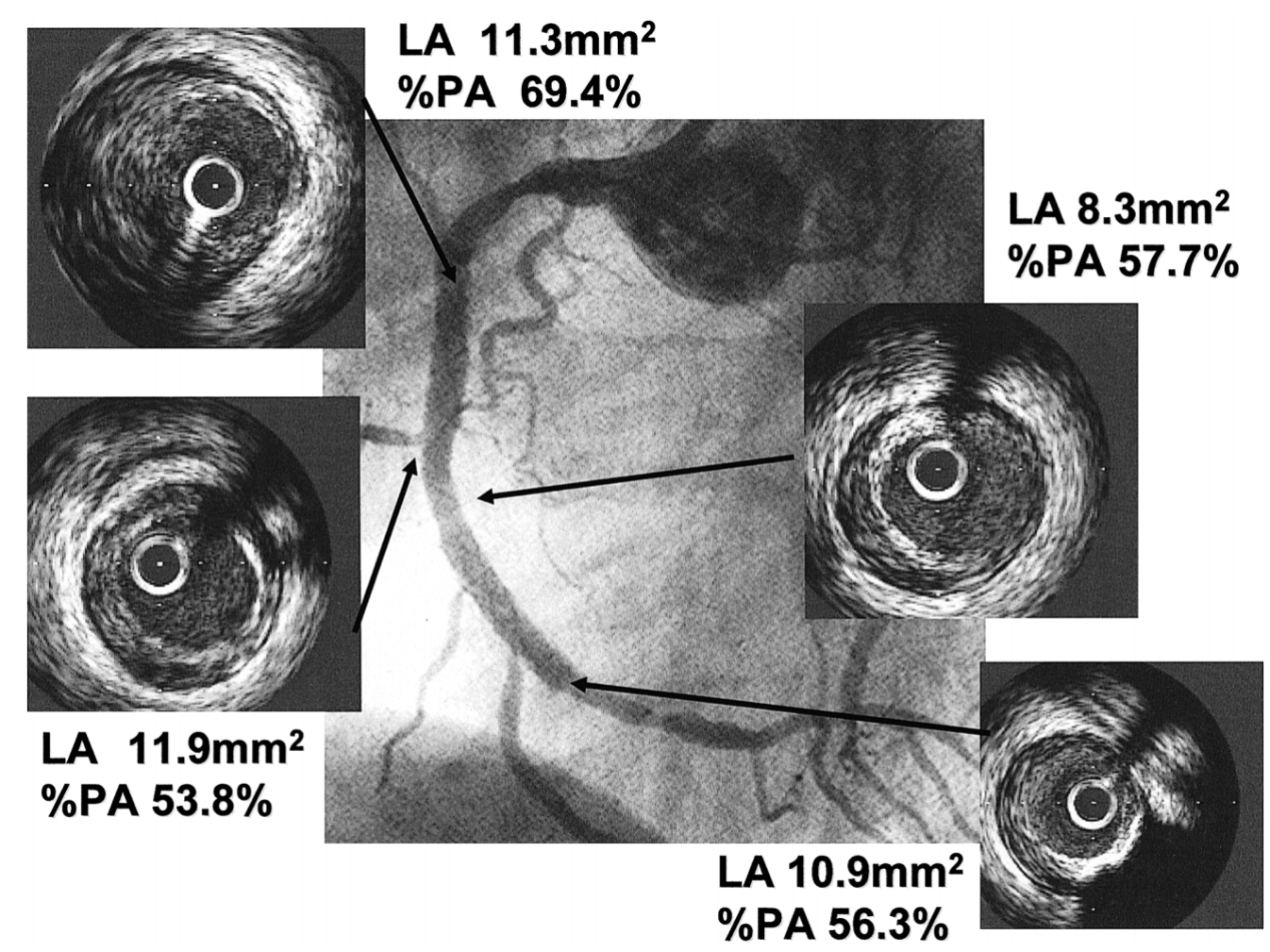

Fig 4. Post-procedural intravascular ultrasound of the case of total-occlusion-type in-stent restenosis shows abundant plaque burden even the lumen is clear. LA, lumen area; PA, plaque area. 


\section{Pre proximal edge F/u proximal edge}

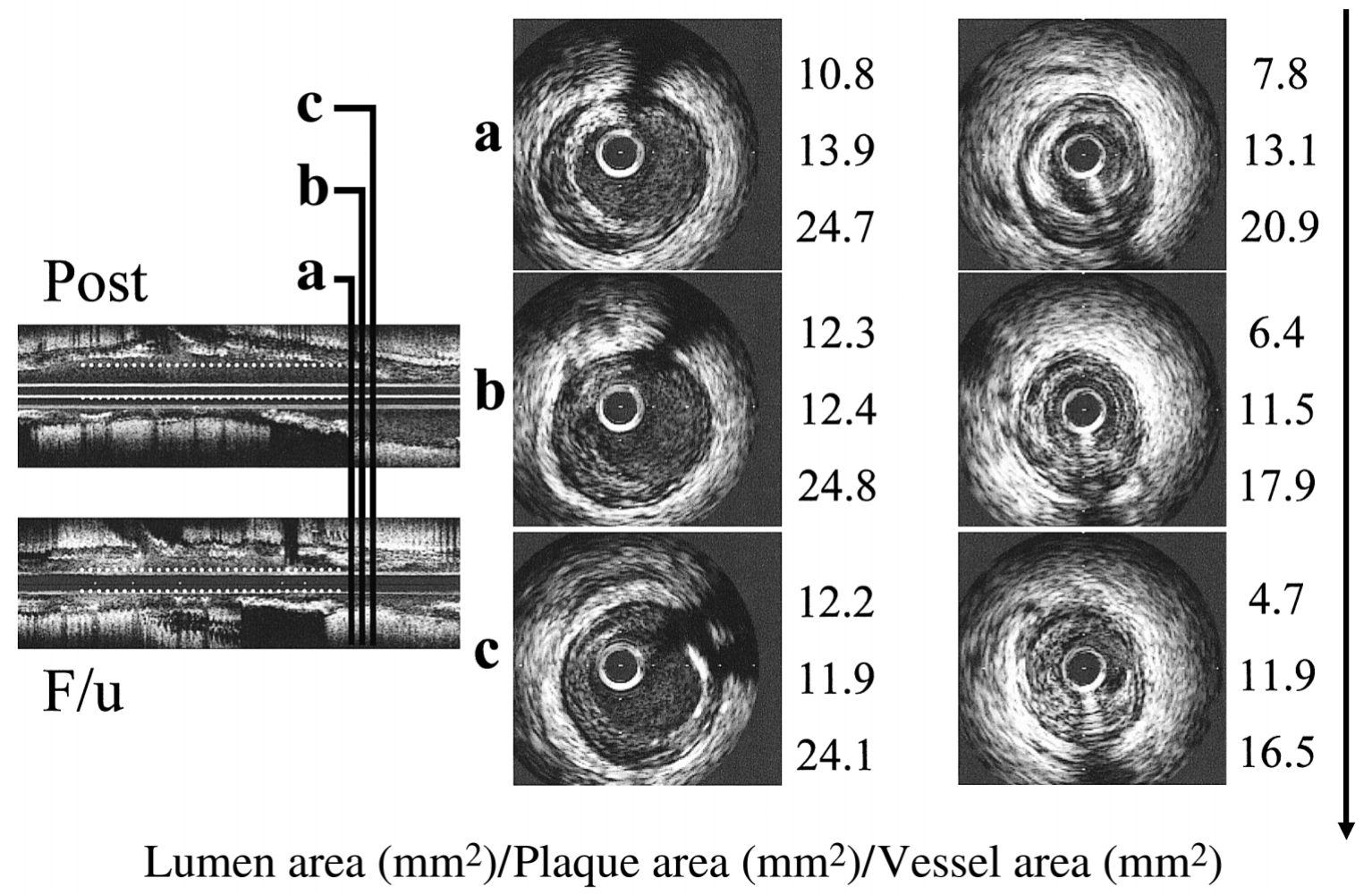

Fig 5. Follow-up (F/u) and post-procedural intravascular ultrasound show lumen narrowing but without an increase in the plaque area.

present study there was 1 case of total-occlusion-type stent restenosis.

\section{Mechanism of Restenosis With DES}

Even though the mechanism of restenosis after DES implantation is difficult to elucidate, our experience in this PK trial lead us to speculate on some likely reasons. In the case of total-occlusion-type restenosis, angiography showed that the SES was fully patent, and its internal side branch was still open, even after the coronary artery was totally occluded. This finding clearly demonstrates the efficacy of the SES in preventing intimal hyperplasia especially within the stent. Therefore, the SES may modify the pattern of restenosis from the diffuse ISR that occurs with BMS implantation to edge restenosis. As a result, the proportion of focal-type restenosis to all restenosis cases might increase with more widespread use of SES. In a serial IVUS study of Palmaz-Schatz stents performed by Hoffmann et al, the dominant periprocedural predictor of stent margin restenosis was the plaque burden of the adjacent reference segments. Although it has not been demonstrated that plaque burden at the stent edge is also a predictor for edge restenois with DES, in the present cases of restensosis with a SES, the post-procedural \%PA adjacent to the proximal edge was more than $50 \%$, even though the lumen was well preserved. Thus, our findings suggest that incomplete coverage by the SES may be involved in the occurrence of stent-edge restenosis. Because it is unclear what \%PA is small enough to prevent the restensosis, accumulation of more data is required. Another potential explanation is procedural factors such as geographic miss. Regarding the edge effect of a radioactive stent, Albiero et $\mathrm{al}^{9}$ suggested that it arises from a combination of 2 factors: (1) a low dose of radiation at the stent margin and (2) an aggressive approach to stenting as indicated by a high balloon-to-artery ratio. Angiolillo et al also suggested that radioactive stents and DES share many common features and that lessons learned from intracoronary brachytherapy should be applied to the DES era10 They suggested that axial geographic misses because of inadequate stent size may be another reason for the edge effect.

It is difficult to elucidate the mechanism for the margin stenosis shown in Fig 3. Because significant intimal hyperplasia (increase in PA during follow-up) was not observed at the stent edge, it is possible that vessel narrowing is associated with this angiographic finding and would be consistent with a recently published report that attributed remodeling to edge restenosis after SES implantation.1 Such vessel narrowing could contribute to insufficient coronary flow. Another explanation is that this vessel shrinkage represents an allergic reaction, as Virmani et al ${ }^{12}$ suggested. However, this is no more than speculation and it is apparent that a variety of factors are involved in margin stenosis.

\section{Clinical Implications}

Since the initial report of the Sirius trial, much attention has been paid to edge restenosis after DES deployment and as a result, an optimal stenting technique for DES has been recommended: (1) avoid aggressive predilatation, (2) avoid post-dilatation outside the stent, (2) full coverage of the lesion, and (3) no gap between stents, 13,14 Our results from the PK trial indicate that procedural factors, especially stent length, also could be important in determining the longterm outcome. 


\section{Study Limitations}

Stent thrombosis was not a complication in this study, which suggests the efficacy and safety of ticlopidine in preventing stent thrombosis in the setting of SES implantation. However, this was an observational study of a small number of subjects. Long-term follow-up data are required to confirm the durability of this type of stent. Additionally, the long-term outcomes may have been affected by the study protocol in which the available sizes and number of stents was limited.

\section{Conclusion}

Sirolimus-coated BX Velocity stents are safe and feasible to use in Japanese patients with coronary artery disease. Neointimal growth within the stent was completely inhibited at 8 months after PCI. However, edge restenosis may occur under some conditions and the mechanisms of this phenomenon remains unclear. Subsequent investigation and additional information are required to better understand the mechanism of restenosis with SES deployment.

\section{References}

1. Morice MC, Serruys PW, Sousa JE, Fajadet J, Hayashi EB, Perin M, et al. A randomized comparison of a sirolimus-eluting stent with a standard stent for coronary revascularization. N Engl J Med 2002; 346: $1773-1780$

2. Moses JW, Leon MB, Popma JJ, Fitzgerald PJ, Holmes DR, O'Shaughnessy C, et al. Sirolimus-eluting stents versus standard stents in patients with stenosis in a native coronary artery. $N$ Engl J Med 2003; 349: 1315-1323.

3. Moussa I, Leon MB, Baim DS, O'Neill WW, Popma JJ, Buchbinder $\mathrm{M}$, et al. Impact of sirolimus-eluting stents on outcome in diabetic patients: A SIRIUS (SIRolImUS-coated Bx Velocity balloon-expandable stent in the treatment of patients with de novo coronary artery lesions) substudy. Circulation 2004; 109: 2273-2278.

4. Colombo A, Moses JW, Morice MC, Ludwing J, Holmes DR,
Spanos V Jr, et al. Randomized study to evaluate sirolimus-eluting stents implanted at coronary bifurcation lesions. Circulation 2004; 109: $1244-1249$.

5. Holmes DR Jr, Leon MB, Moses JW, Popma JJ, Cutlip D, Fitzgerald PJ, et al. Analysis of 1-year clinical outcomes in the SIRIUS trial: A randomized trial of a sirolimus-eluting stent versus a standard stent in patients at high risk for coronary restenosis. Circulation 2004; 109: 634-640.

6. Schampaert E, Cohen EA, Schluter M, Reeves F, Traboulsi M, Title LM, et al. The Canadian study of the sirolimus-eluting stent in the treatment of patients with long de novo lesions in small native coronary arteries (C-SIRIUS). J Am Coll Cardiol 2004; 43: 1110-1115.

7. Otsuka Y, Nakamura M, Yasuda S, Kozuma K, Hara H, Morii I, et al. Comparison of pharmacokinetics of the sirolimus-eluting stent in Japanese patients with those in American patients. J Cardiovasc Pharmacol 2005 (in press).

8. Hoffman R, Mintz GS, Dussaillant GR, Popma JJ, Pichard AD, Satler LF, et al. Patterns and mechanism of in-stent restenosis: A serial intravascular ultrasound study. Circulation 1996; 94: 1247-1254.

9. Alberio R, Nishida T, Adamian M, Amato A, Vaghetti M, Corvaja $\mathrm{N}$, et al. Edge restenosis after implantation of high-activity 32P radioactive-emtting stents. Circulation 2000; 101: 2454-2457.

10. Angiolollo DJ, Sabata M, Alfonso F, Macaya C. "Candy wrapper" effect after drug-eluting stent implantation: Déjà vu or stumbling over the same stone again. Catheter Cardiovasc Interv 2004; 61: $387-$ 391.

11. Virmani R, Guaglium G, Farb A, Musumeci G, grieco N, Motta T, et al. Localized hypersensitivity and late coronary thrombosis secondary to a sirolimus-eluting stent: Should we be cautious? Circulation 2004; 109: 701-705.

12. Takebayashi H, Kobayashi Y, Mintz GS, Carlier SG, Fujii K, Yasuda $\mathrm{T}$, et al. Intravascular ultrasound assessment of lesions with target vessel failure after sirolimus-eluting stent implantation. Am J Cardiol 2005; 95: 498-502.

13. Lemos PA, Saia F, Lighthart JMR, Arampatzis CA, Sianos G, Tanabe K, et al. Coronary restenosis after sirolimus-eluting stent implantation: Morphological description and mechanistic analysis from a consecutive series of cases. Circulation 2003; 108: 257-260.

14. Colombo A, Orlic D, Stankovic G, Corvaja N, Spanos V, Montorfano $\mathrm{M}$, et al. Preliminary observations regarding angiographic pattern of restenosis after rapamycin-eluting stent implantation. Circulation 2003; 107: 2178-2180. 